TRANSACTIONS OF THE

AMERICAN MATHEMATICAL SOCIETY

Volume 363, Number 4, April 2011, Pages 1699-1719

S 0002-9947(2010)05343-7

Article electronically published on November 1, 2010

\title{
WEIGHTED INEQUALITIES FOR THE TWO-DIMENSIONAL ONE-SIDED HARDY-LITTLEWOOD MAXIMAL FUNCTION
}

\author{
L. FORZANI, F. J. MARTÍN-REYES, AND S. OMBROSI
}

\begin{abstract}
In this work we characterize the pairs of weights $(w, v)$ such that the one-sided Hardy-Littlewood maximal function in dimension two is of weaktype $(p, p), 1 \leq p<\infty$, with respect to the pair $(w, v)$. As an application of this result we obtain a generalization of the classic Dunford-Schwartz Ergodic Maximal Theorem for bi-parameter flows of null-preserving transformations.
\end{abstract}

\section{InTRODUCTION AND MAIN RESULtS}

The study of the one-sided Hardy-Littlewood maximal function

$$
M^{+} f(x)=\sup _{h>0} \frac{1}{h} \int_{x}^{x+h}|f|,
$$

defined for measurable functions $f: \mathbb{R} \rightarrow \mathbb{R}$ started in the 1930s. During the same years the basic results about the ergodic maximal operator were obtained. If $(X, \mathcal{F}, \mu)$ is a measure space and $\left\{\tau^{t}: t \in \mathbb{R}\right\}$ is a flow of measure-preserving transformations on $X$, the ergodic maximal function is defined by

$$
M_{\tau} f(x)=\sup _{h>0} \frac{1}{h} \int_{0}^{h}\left|f\left(\tau^{t} x\right)\right| d t
$$

for all measurable functions $f: X \rightarrow \mathbb{R}$. We notice that $M^{+}$is a particular case of the ergodic maximal operator since $M^{+}=M_{\tau}$ when $(X, \mu)$ is $\mathbb{R}$ with the Lebesgue measure and $\tau^{t}(x)=x+t$. Nowadays it is well known that, by transference arguments, the results of the boundedness for the general operator $M_{\tau}$ can be obtained by the corresponding results for the particular case $M^{+}$(see [18] for a recent exposition in the discrete case).

Although the search started with the one-sided Hardy-Littlewood maximal operator, we notice that in harmonic analysis the usual Hardy-Littlewood maximal

Received by the editors May 28, 2007.

2010 Mathematics Subject Classification. Primary 42B25; Secondary 47A35, 37A40.

Key words and phrases. Weights, one-sided maximal function, ergodic maximal theorem.

The research of the first author has been partially supported by CONICET, grant PIP 5810, and the Universidad Nacional del Litoral.

The research of the second author has been partially supported by the Junta de Andalucía, grants FQM-354 and the FQM-01509, and the Spanish government, grants MTM2005-08350-C0302 and MTM2008-06621-C02-02.

The research of the third author has been partially supported by the Universidad Nacional del Sur, grant SGCyT-UNS PGI 24/L058, and by a grant of the Spanish government, MEC Res. $26 / 05 / 2006$.

(C)2010 American Mathematical Society Reverts to public domain 28 years from publication 
operator is the two-sided operator

$$
M f(x)=\sup _{h>0} \frac{1}{2 h} \int_{x-h}^{x+h}|f| .
$$

In $\mathbb{R}^{n}$, for $n \geq 1$, the Hardy-Littlewood maximal operator is defined by

$$
M f(x)=\sup _{h>0} \frac{1}{|Q(x, h)|} \int_{Q(x, h)}|f|,
$$

where $Q(x, h)$ denotes the cube of center $x$, sides parallel to the axis, and side of length $2 h$. This operator has been extensively studied. In particular Muckenhoupt [12] (see also [4) established necessary and sufficient conditions on a positive function (weight) $w$ for the inequality

$$
\int_{\mathbb{R}^{n}}(M f)^{p} w \leq C \int_{\mathbb{R}^{n}}|f|^{p} w, \quad 1<p<\infty,
$$

to hold for all measurable functions with a constant independent of $f$. Muckenhoupt also solved the same problem for the weak type inequality

$$
\int_{\left\{x \in \mathbb{R}^{n}: M f(x)>\lambda\right\}} w \leq \frac{C}{\lambda^{p}} \int_{\mathbb{R}^{n}}|f|^{p} v .
$$

Since then a lot of work has been done establishing the same kind of inequalities for other operators. Surprisingly, it took fourteen years until E. Sawyer 17] characterized the good weights for the one-sided Hardy-Littlewood maximal operator in the real line. These results were applied to the ergodic setting (for instance see [11, [14, 2, 8]). We remark that weighted inequalities for other one-sided operators have been studied before and after the seminal work of Muckenhoupt. Examples of these operators are the averaging Hardy operator [1]

$$
T f(x)=\frac{1}{x} \int_{0}^{x} f
$$

and the Liouville fractional integral operator

$$
T f(x)=\int_{x}^{\infty}(t-x)^{\alpha} f(t) d t .
$$

We remark that these one-sided operators are defined in the real line.

E. Sawyer [16] studied the weighted inequalities for a one-sided operator in $\mathbb{R}^{2}$. More precisely, he considered the two-dimensional Hardy operator defined as $H f(x, y)=\int_{0}^{x} \int_{0}^{y} f(s, t) d s d t$ for $x, y>0$. As Sawyer mentioned in his paper, the proofs do not generalize (at least in an obvious way) to higher dimensions. Until now, the result has not been extended to $\mathbb{R}^{n}$ with $n \geq 3$.

But what can be said about the one-sided Hardy-Littlewood maximal operator in $\mathbb{R}^{n}$ for $n>1$ ? It is quite clear that a possible and natural definition is

$$
M^{+} f(x)=\sup _{h>0} \frac{1}{h^{n}} \int_{x_{1}}^{x_{1}+h} \cdots \int_{x_{n}}^{x_{n}+h}|f|,
$$


where $x=\left(x_{1}, \ldots, x_{n}\right)$. Once we have fixed the operator, we settle the question of finding necessary and sufficient conditions for the weighted inequalities

$$
\begin{gathered}
\int_{\mathbb{R}^{n}}\left(M^{+} f\right)^{p} w \leq C \int_{\mathbb{R}^{n}}|f|^{p} w, \\
\int_{\left\{x \in \mathbb{R}^{n}: M^{+} f(x)>\lambda\right\}} w \leq \frac{C}{\lambda^{p}} \int_{\mathbb{R}^{n}}|f|^{p} v
\end{gathered}
$$

to hold. As far as we know, this problem has not been solved; an early discussion can be found in [10] and the characterization of the weak type of a one-sided dyadic maximal operator in $\mathbb{R}^{n}$ appears in [13. However, the usual nondyadic case seems to be more complicated, and therefore it requires a deeper analysis.

In this paper we answer the question associated with the weighted weak type inequality of $M^{+}$in dimension two. That is, we show a characterization of the pairs of weights $(w, v)$ such that the operator $M^{+}$in $\mathbb{R}^{2}$ is of weak type $(p, p)$ with respect to the pair $(w, v)$. The conditions in the weights are the expectable geometric conditions similar to the conditions of the classes of Muckenhoupt $A_{p}\left(\mathbb{R}^{2}\right)$.

In order to state the main result of this paper we need to introduce some notation.

If $Q=[a-h, a] \times[b-h, b]$ is a square with sides parallel to the axis, we set $Q^{+}=[a, a+h] \times[b, b+h]$. Now, we define the one-sided Muckenhoupt conditions in $\mathbb{R}^{2}$.

Definition 1.1. Let $(w, v)$ be a pair of nonnegative measurable functions on $\mathbb{R}^{2}$. Let $1<p<\infty$ and let $p^{\prime}$ be its conjugate exponent, that is, $p+p^{\prime}=1$. It is said that $(w, v)$ satisfies $A_{p}^{+}\left(\mathbb{R}^{2}\right)$, or $(w, v) \in A_{p}^{+}\left(\mathbb{R}^{2}\right)$, if there exists a positive constant $C$ such that for all squares $Q$

$$
\left(\frac{1}{|Q|} \int_{Q} w\right)^{1 / p}\left(\frac{1}{\left|Q^{+}\right|} \int_{Q^{+}} v^{1-p^{\prime}}\right)^{1 / p^{\prime}} \leq C .
$$

It is said that $(w, v)$ satisfies $A_{1}^{+}\left(\mathbb{R}^{2}\right)$ if there exists a positive constant $C$ such that for all $h>0$

$$
\frac{1}{h^{2}} \int_{x_{1}-h}^{x_{1}} \int_{x_{2}-h}^{x_{2}} w \leq C v(x) \text { for almost every } x=\left(x_{1}, x_{2}\right) .
$$

$A_{p}^{+}\left(\mathbb{R}^{2}\right)$ is similar to the Muckenhoupt $A_{p}\left(\mathbb{R}^{2}\right)$ condition. We recall that $(w, v)$ satisfies $A_{p}\left(\mathbb{R}^{2}\right), 1<p<\infty$, if there exists a positive constant $C$ such that for all squares $Q$

$$
\left(\frac{1}{|Q|} \int_{Q} w\right)^{1 / p}\left(\frac{1}{|Q|} \int_{Q} v^{1-p^{\prime}}\right)^{1 / p^{\prime}} \leq C .
$$

It is said that $(w, v)$ satisfies $A_{1}\left(\mathbb{R}^{2}\right)$ if there exists a positive constant $C$ such that for all squares $Q$

$$
\frac{1}{|Q|} \int_{Q} w \leq C v(x) \text { for almost every } x \in Q .
$$

It is easy to see that if $(w, v)$ satisfies the classic Muckenhoupt condition $A_{p}\left(\mathbb{R}^{2}\right)$ and if $g$ is a nonnegative function on $\mathbb{R}^{2}$ which is nondecreasing on each variable separately, then $(g w, g v) \in A_{p}^{+}\left(\mathbb{R}^{2}\right)$. In particular, $(g, g) \in A_{1}^{+}\left(\mathbb{R}^{2}\right)$.

Now we are ready to state the main theorem of the paper. 
Theorem 1.2. Let $(w, v)$ be a pair of nonnegative measurable functions on $\mathbb{R}^{2}$. Let $1 \leq p<\infty$. Then, the following conditions are equivalent:

(a) $(w, v) \in A_{p}^{+}\left(\mathbb{R}^{2}\right)$.

(b) There is a constant $C$ such that for every measurable function $f$ and every $\lambda>0$ the inequality

$$
w\left(\left\{x: M^{+} f(x)>\lambda\right\}\right) \leq C \lambda^{-p} \int_{\mathbb{R}^{2}}|f|^{p} v
$$

holds, where $w(E)$ stands for $\int_{E} w$.

The proof is geometric and is based on Lemma 3.1 which is a covering lemma. The search for this lemma has been inspired by the covering arguments in [13]. It is not clear to us if Lemma 3.1 can be extended to higher dimensions.

We already mentioned that if $w$ is a nonnegative function on $\mathbb{R}^{2}$ which is nondecreasing on each variable separately, then $(w, w) \in A_{1}^{+}\left(\mathbb{R}^{2}\right)$. Therefore, we have the following corollary.

Corollary 1.3. If $w$ is a nonnegative function on $\mathbb{R}^{2}$ which is increasing (nondecreasing) on each variable separately, then

$$
\int_{\{x: M+f(x)>\lambda\}} w \leq \frac{C}{\lambda} \int_{\mathbb{R}^{n}}|f| w
$$

for all $\lambda>0$ and all measurable function $f$.

Actually, we want to point out that this corollary follows easily from the DunfordSchwartz ergodic maximal theorem. In the next theorem we recall the result by Dunford and Schwartz.

Theorem 1.4 ([6, Lemma VIII.7.11]). Let $(X, \mathcal{F}, \mu)$ be a measure space and let $\mathcal{T}=\left\{T^{t}: t=\left(t_{1}, \ldots, t_{n}\right), t_{1}, \ldots, t_{n}>0\right\}$ be a strongly measurable semi-group of operators in $L^{1}(X, \mathcal{F}, \mu)$ with $\left\|T^{t}\right\|_{1} \leq 1$ and $\left\|T^{t}\right\|_{\infty} \leq 1$. Let

$$
M_{\mathcal{T}} f(x)=\sup _{h>0} \frac{1}{h^{n}}\left|\int_{0}^{h} \ldots \int_{0}^{h} T^{t} f(x) d t_{1} \ldots d t_{n}\right| .
$$

Then there is an absolute constant $C_{n}$, which is independent of the semi-group and independent of $f$, such that

$$
\mu\left(\left\{x \in X: M_{\mathcal{T}} f(x)>\lambda\right\}\right) \leq \frac{1}{C_{n} \lambda} \int_{\left\{x \in X: M_{\mathcal{T}} f(x)>C_{n} \lambda\right\}}|f| d \mu
$$

for all $\lambda>0$.

Observe that if $w$ is a nonnegative function in $\mathbb{R}^{n}$ which is increasing on each variable separately, then the semigroup of operators $T^{t} f(x)=f(x+t), t \in \mathbb{R}^{n}$, $t=\left(t_{1}, \ldots, t_{n}\right), t_{i}>0$, is a contraction in $L^{1}(w)$ and in $L^{\infty}(w)$. Therefore, we can apply the Dunford-Schwartz ergodic theorem, and Corollary 1.3 follows not only in $\mathbb{R}^{2}$ but in $\mathbb{R}^{n}$ for all $n$. This result seems to not be well known, and the authors have not found it in the literature.

We point out that we do not know any geometric proof of the Dunford-Schwartz ergodic theorem. However, Theorem 1.2 gives a geometric proof of Corollary 1.3 , which is the Dunford-Schwartz ergodic theorem for the semigroup $T^{t} f(x)=f(x+t)$ in $\mathbb{R}^{2}$. 
As an application in ergodic theory of our main result, we obtain a theorem which is in some sense an extension of the Dunford-Schwartz ergodic theorem. In order to state it, consider a $\sigma$-finite measure space $(X, \mathcal{F}, \mu)$ and let $\left\{\tau^{t}: t \in \mathbb{R}^{2}\right\}$ be a bi-parameter flow of null-preserving transformations on $X$. That is,

(a) For all $t \in \mathbb{R}^{2}, \tau^{t}: X \rightarrow X$ is a measurable map such that if $\mu(E)=0$, then $\mu\left(\tau^{t} E\right)=0$.

(b) The map $(t, x) \rightarrow \tau^{t}(x)$ from $\mathbb{R}^{2} \times X \rightarrow X$ is measurable with respect to the completion of the $\sigma$-algebra product in $\mathbb{R}^{2} \times X$.

(c) $\tau^{0}(x)=x$ for all $x \in X$ and $\tau^{t} \circ \tau^{s}=\tau^{t+s}$ for all $t, s \in \mathbb{R}^{2}$.

The flow induces a group $\mathcal{T}=\left\{T^{t}: t \in \mathbb{R}^{2}\right\}$ of operators acting on measurable functions and defined by

$$
T^{t} f(x)=f\left(\tau^{t} x\right) .
$$

For each $h>0$ we consider the averages over squares

$$
A_{h} f(x)=\frac{1}{h^{2}} \int_{0}^{h} \int_{0}^{h} T^{t} f(x) d t .
$$

To study the convergence of $A_{h}$ as $h \rightarrow+\infty$, the usual thing is to consider the ergodic maximal operator

$$
M_{\mathcal{T}} f(x)=\sup _{h>0}\left|A_{h} f(x)\right|
$$

By using the Dunford-Schwartz theorem quoted in the introduction, we have that if each $T^{t}$ is a contraction in $L^{1}(\mu)$, then the maximal operator $M_{\mathcal{T}}$ is of weak type $(1,1)$, that is, there exists $C$ such that

$$
\mu\left(\left\{x \in X: M_{\mathcal{T}} f(x)>\lambda\right\}\right) \leq \frac{C}{\lambda} \int_{X}|f| d \mu
$$

for all $\lambda>0$ and all $f \in L^{1}(\mu)$. Our result in ergodic theory, Theorem 1.5, states that (2) holds under the assumption that the group $\mathcal{T}$ is Cesàro bounded in $L^{1}(\mu)$, which means that there exists $C>0$ such that

$$
\sup _{h>0} \int_{X}\left|A_{h} f\right| \leq C \int_{X}|f| d \mu
$$

for all measurable $f \geq 0$. Now we are ready to state the theorem.

Theorem 1.5. Let $(X, \mathcal{F}, \mu)$ be a $\sigma$-finite measure space and let $\left\{\tau^{t}: t \in \mathbb{R}^{2}\right\}$ be a flow of null-preserving transformations on $X$. Assume that the group $\mathcal{T}$ is Cesàro bounded in $L^{1}(\mu)$. Then there is a constant $C>0$ such that

$$
\mu\left(\left\{x \in X: M_{\mathcal{T}} f(x)>\lambda\right\}\right) \leq \frac{C}{\lambda} \int_{X}|f| d \mu
$$

for all $\lambda>0$ and all $f \in L^{1}(\mu)$.

We remark that the assumption about the group, together with the properties of the flow, assures that $A_{h} f$ is defined and (3) holds for all $f \in L^{1}(\mu)$. Notice also that if each $T^{t}$ is a contraction in $L^{1}(\mu)$, then the group $\mathcal{T}$ is Cesàro bounded in $L^{1}(\mu)$. Therefore, our assumption is weaker than the one we need to apply the Dunford-Schwartz theorem (in Final Remarks 5.1 we observe that there are groups which are Cesàro bounded but where the operators are not a contraction in $L^{1}(\mu)$ ).

The paper is organized as follows: $\S 2$ is dedicated to introducing notation, the definitions of some maximal operators, and some results about them; in $\S 3$ and $\S 4$ 
we prove the main result and the covering lemma, respectively; in the last section we prove Theorem 1.5 and make some remarks.

As usual, if $E \subset \mathbb{R}^{n}$ is measurable, $|E|$ denotes the Lebesgue measure of $E$, and if $w$ is a measurable function, then $w(E)=\int_{E} w$. Throughout the paper, the letter $C$ will denote a positive constant whose value may change from line to line.

\section{NotATion AND DEFINitions}

If $I=[a, b]$ is a bounded interval we denote $I^{+}=[b, 2 b-a]$ and $I^{-}=[2 a-b, a]$. By a square we mean a square with sides parallel to the axis. If $Q=I_{1} \times I_{2}$ is a square, then $l(Q)$ stands for the length of the side of $Q$, that is, the length of $I_{1}$ or $I_{2}$, and we denote the squares $I_{1}^{+} \times I_{2}^{+}$, and $I_{1}^{-} \times I_{2}^{-}$by $Q^{+}$and $Q^{-}$, respectively. We shall say that a square $Q$ is of dyadic size if $l(Q)=2^{k}$ for some $k \in \mathbb{Z}$. If $Q$ is a square and $\alpha$ is a positive number, $\alpha Q$ is the square with the same center as $Q$ and $l(\alpha Q)=\alpha l(Q)$. If $Q=[a, a+h] \times[b, b+h]$, then $\widetilde{Q}$ is the dilation of $Q$ to the right and to the bottom in half the length of the side of $Q$, that is, $\widetilde{Q}=\left[a, a+\frac{3}{2} h\right] \times\left[b-\frac{h}{2}, b+h\right]$. See Figure 1 .

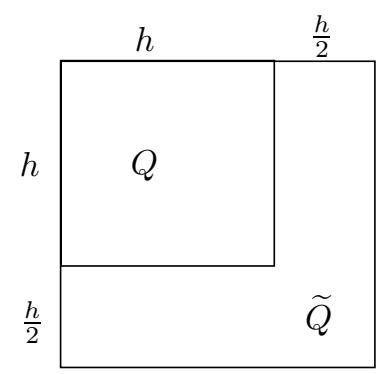

Figure 1. $Q$ and $\widetilde{Q}$.

Let $x \in \mathbb{R}^{2}, x=\left(x_{1}, x_{2}\right)$, and let $h$ be a positive real number. We denote $Q_{x, h}=\left[x_{1}, x_{1}+h\right] \times\left[x_{2}, x_{2}+h\right], Q_{x, h^{-}}=\left[x_{1}-h, x_{1}\right] \times\left[x_{2}-h, x_{2}\right]$. With this notation, we define the maximal functions

$$
M^{+} f(x)=\sup _{h>0} \frac{1}{\left|Q_{x, h}\right|} \int_{Q_{x, h}}|f| \quad \text { and } \quad M^{-} f(x)=\sup _{h>0} \frac{1}{\left|Q_{x, h^{-}}\right|} \int_{Q_{x, h^{-}}}|f| .
$$

Now we divide the square $Q_{x, h}$ into four squares (see Figure 2):

$$
\begin{aligned}
Q_{x, h} & =Q_{x, \frac{h}{2}} \cup Q_{x, h}^{1} \cup Q_{x, h}^{2} \cup Q_{x, h}^{3}, \\
Q_{x, h}^{2} & =\left[x_{1}+\frac{h}{2}, x_{1}+h\right] \times\left[x_{2}, x_{2}+\frac{h}{2}\right], \\
Q_{x, h}^{3} & =\left[x_{1}, x_{1}+\frac{h}{2}\right] \times\left[x_{2}+\frac{h}{2}, x_{2}+h\right],
\end{aligned}
$$

and

$$
Q_{x, h}^{1}=\left[x_{1}+\frac{h}{2}, x_{1}+h\right] \times\left[x_{2}+\frac{h}{2}, x_{2}+h\right],
$$




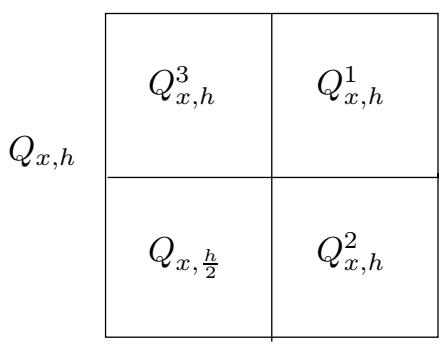

Figure 2. Subsquares.

and we define

$$
\begin{aligned}
M^{+1} f(x) & =\sup _{h>0} \frac{1}{\left|Q_{x, h}^{1}\right|} \int_{Q_{x, h}^{1}}|f|, \\
M^{+2} f(x) & =\sup _{h>0} \frac{1}{\left|Q_{x, h}^{2}\right|} \int_{Q_{x, h}^{2}}|f|, \\
M^{+3} f(x) & =\sup _{h>0} \frac{1}{\left|Q_{x, h}^{3}\right|} \int_{Q_{x, h}^{3}}|f| .
\end{aligned}
$$

We have that $M^{+}$is essentially equivalent to the sum of the maximal operators $M^{+i}, i=1,2,3$. We state this result in the next proposition.

Proposition 2.1. The following inequality holds for every measurable function:

$$
\begin{aligned}
& \frac{1}{12}\left(M^{+1} f(x)+M^{+2} f(x)+M^{+3} f(x)\right) \\
& \quad \leq M^{+} f(x) \leq \frac{1}{3}\left(M^{+1} f(x)+M^{+2} f(x)+M^{+3} f(x)\right) .
\end{aligned}
$$

Proof. By density arguments it is enough to prove it for functions $f \in L^{1}(d x)$. It is clear that for every $h>0$

$$
\frac{1}{\left|Q_{x, h}^{i}\right|} \int_{Q_{x, h}^{i}}|f| \leq 4 \frac{1}{\left|Q_{x, h}\right|} \int_{Q_{x, h}}|f| \leq 4 M^{+} f(x) .
$$

Therefore $M^{+1} f(x)+M^{+2} f(x)+M^{+3} f(x) \leq 12 M^{+} f(x)$. On the other hand, if $h>0$ we have

$$
\begin{aligned}
\frac{1}{\left|Q_{x, h}\right|} \int_{Q_{x, h}}|f| & =\frac{1}{\left|Q_{x, h}\right|}\left(\int_{Q_{x, \frac{h}{2}}}|f|+\int_{Q_{x, h} \backslash Q_{x, \frac{h}{2}}}|f|\right) \\
& \leq \frac{1}{4\left|Q_{x, \frac{h}{2}}\right|} \int_{Q_{x, \frac{h}{2}}}|f|+\frac{1}{4}\left(M^{+1} f(x)+M^{+2} f(x)+M^{+3} f(x)\right) \\
& \leq \frac{1}{4}\left(M^{+} f(x)+M^{+1} f(x)+M^{+2} f(x)+M^{+3} f(x)\right) .
\end{aligned}
$$

Taking supremum on $h>0$,

$$
M^{+} f(x) \leq \frac{1}{4}\left(M^{+} f(x)+M^{+1} f(x)+M^{+2} f(x)+M^{+3} f(x)\right) .
$$


Since $f \in L^{1}$ we have $M^{+} f(x)<\infty$ a.e., and therefore

$$
M^{+} f(x) \leq \frac{1}{3}\left(M^{+1} f(x)+M^{+2} f(x)+M^{+3} f(x)\right) .
$$

For technical reasons, in the proof of the main result we shall use the maximal operator $\mathcal{M}^{+}$defined by

$$
\mathcal{M}^{+} f(x)=\sup _{k \in \mathbb{Z}} \frac{1}{\left|Q_{x, 2^{k}}\right|} \int_{Q_{x, 2^{k}}}|f|,
$$

that is, we only take cubes $Q_{x, h}$ of dyadic size. This operator is essentially equivalent to $M^{+}$. In fact,

$$
\frac{1}{4} M^{+} \leq \mathcal{M}^{+} \leq M^{+} .
$$

We also consider $\mathcal{M}^{+i}, i=1,2,3,4$, defined by

$$
\mathcal{M}^{+i} f(x)=\sup _{k \in \mathbb{Z}} \frac{1}{\left|Q_{x, 2^{k}}^{i}\right|} \int_{Q_{x, 2^{k}}^{i}}|f| .
$$

The operators $\mathcal{M}^{+}$and $\mathcal{M}^{+i}$ are related in the same way as $M^{+}$and $M^{+i}$. We establish this relation in the next proposition.

Proposition 2.2. The following inequality holds for every measurable function:

$$
\begin{gathered}
\frac{1}{12}\left(\mathcal{M}^{+1} f(x)+\mathcal{M}^{+2} f(x)+\mathcal{M}^{+3} f(x)\right) \leq \mathcal{M}^{+} f(x) \\
\quad \leq \frac{1}{3}\left(\mathcal{M}^{+1} f(x)+\mathcal{M}^{+2} f(x)+\mathcal{M}^{+3} f(x)\right) .
\end{gathered}
$$

The proof is the same as the proof of Proposition 2.1.

\section{Proof of Theorem 1.2}

The relevant implication is $(a) \Longrightarrow(b)$ since $(b) \Longrightarrow(a)$ follows as in the classic case of Muckenhoupt weights, and we omit it.

$(a) \Longrightarrow(b)$. Since $M^{+}$and $\mathcal{M}^{+}$are essentially equivalent, it is enough to prove (b) for the operator $\mathcal{M}^{+}$. That is, we are going to prove the following inequality:

$$
w\left(\left\{x: \mathcal{M}^{+} f(x)>\lambda\right\}\right) \leq C \lambda^{-p} \int_{\mathbb{R}^{2}}|f|^{p} v .
$$

Observe that (4) follows from the inequality

$$
w\left(\left\{x: \lambda<\mathcal{M}^{+} f(x) \leq 2 \lambda\right\}\right) \leq \frac{C}{\lambda^{p}} \int_{\mathbb{R}^{2}}|f|^{p} v .
$$

In fact, if (5) holds, then

$$
\begin{aligned}
w\left(\left\{x: \mathcal{M}^{+} f(x)>\lambda\right\}\right) & =\sum_{k=0}^{\infty} w\left(\left\{x: 2^{k} \lambda<\mathcal{M}^{+} f(x) \leq 2^{k+1} \lambda\right\}\right) \\
& \leq \sum_{k=0}^{\infty} \frac{C}{2^{p k} \lambda^{p}} \int_{\mathbb{R}^{2}}|f|^{p} v=\frac{2^{p} C}{\left(2^{p}-1\right) \lambda^{p}} \int_{\mathbb{R}^{2}}|f|^{p} v .
\end{aligned}
$$


Proof of (5). By Proposition 2.2, we only have to prove that

(6) $\quad w\left(\left\{x: \lambda<\mathcal{M}^{+i} f(x), \mathcal{M}^{+} f(x) \leq 2 \lambda\right\}\right) \leq \frac{C}{\lambda^{p}} \int_{\mathbb{R}^{2}}|f|^{p} v \quad$ for $i=1,2,3$,

with a constant independent of $f$ and $\lambda$. We shall prove it for $i=2 ; i=1$ and $i=3$ are similar.

Proof of (6) for $i=2$. Let us consider for each $\xi>0$ the truncated maximal operator

$$
\mathcal{M}_{\xi}^{+2} f(x)=\sup _{h=2^{k}>\xi, k \in \mathbb{Z}} \frac{4}{h^{2}} \int_{Q_{x, h}^{2}}|f| .
$$

Since $\mathcal{M}_{\xi}^{+2} f \uparrow \mathcal{M}^{+2} f$ as $\xi \downarrow 0^{+}$, it follows from the monotone convergence theorem that it suffices to prove that

$$
w\left(\left\{x: \lambda<\mathcal{M}_{\xi}^{+2} f(x), \mathcal{M}^{+} f(x) \leq 2 \lambda\right\}\right) \leq \frac{C}{\lambda^{p}} \int_{\mathbb{R}^{2}}|f|^{p} v
$$

for all $\lambda>0$ and all measurable $f$ with a constant independent of $\xi, \lambda$ and $f$.

To prove (7) we shall need the following covering lemma which is the key result of this paper. Notice that we need similar but different lemmas if we are dealing with $\mathcal{M}^{+i}, i=1,3$, instead of $\mathcal{M}^{+2}$.

Lemma 3.1. Let $f$ be a nonnegative measurable function. Let $A=\left\{x_{j}, j=\right.$ $1, \ldots, n\}$ be a finite set of points in $\mathbb{R}^{2}$. Assume that for each $x_{j} \in A$ we have an associated square $Q_{j}$ of dyadic size such that its upper right corner is $x_{j}$ and

$$
\frac{1}{\left|Q_{j}\right|} \int_{Q_{j}^{+2}} f>\frac{\lambda}{4}
$$

Then there exists a set $\Gamma \subset\{1, \ldots, n\}$ such that if $\widetilde{Q_{j}}$ is the dilation of $Q_{j}$ to the right and to the bottom in half the length of the side of $Q_{j}$, we have

$$
A \subset \bigcup_{i \in \Gamma} \widetilde{Q_{i}}
$$

and

$$
\frac{1}{\left|Q_{j}\right|} \int_{\left(\widetilde{Q_{j}}\right)^{+}} f>\frac{\lambda}{4}
$$

Moreover, $\widetilde{Q_{i}} \nsubseteq \widetilde{Q_{j}}, i \neq j, i, j \in \Gamma$, and the squares $\widetilde{Q_{i}}, i \in \Gamma$, of the same size are almost disjoint. That is, there exists a constant $C$ such that for all $l$

$$
\sum_{\left\{i \in \Gamma: l\left(Q_{i}\right)=l\right\}} \chi_{\widetilde{Q_{i}}} \leq C .
$$

(Consequently, the squares $\left(\widetilde{Q_{i}}\right)^{+}$with $i \in \Gamma$ of the same size are almost disjoint too.) Further, if

$$
\frac{1}{\left|Q_{j}\right|} \int_{\left(\widetilde{Q_{j}}\right)^{+}} f \leq 8 \lambda,
$$

then there exists a family of sets $\left\{F_{j}\right\}_{j \in \Gamma}$ with $F_{j} \subset\left(\widetilde{Q_{j}}\right)^{+}$such that

$$
\frac{\lambda}{8}<\frac{1}{\left|Q_{j}\right|} \int_{F_{j}} f
$$


and they are almost disjoint, i.e, there exists $C$ (independent of everything) such that

$$
\sum_{j \in \Gamma} \chi_{F_{j}}(x) \leq C
$$

We postpone the proof of the lemma to the next section.

Proof of (77). Observe first that if $\left(w_{i}, v_{i}\right) \in A_{p}^{+}\left(\mathbb{R}^{2}\right), i=1,2$, then

$\left(\max \left\{w_{1}, w_{2}\right\}, \max \left\{v_{1}, v_{2}\right\}\right) \in A_{p}^{+}\left(\mathbb{R}^{2}\right)$ and $\left(\min \left\{w_{1}, w_{2}\right\}, \min \left\{v_{1}, v_{2}\right\}\right) \in A_{p}^{+}\left(\mathbb{R}^{2}\right)$.

In particular, for each $n \in \mathbb{N}$, if $(w, v) \in A_{p}^{+}\left(\mathbb{R}^{2}\right)$, then the pairs $\left(w_{n}, v_{n}\right)$ and $\left(\widetilde{w}_{n}, \widetilde{v}_{n}\right)$ belong to $A_{p}^{+}\left(\mathbb{R}^{2}\right)$ with a uniform constant, where $w_{n}=\max \left\{w, \frac{1}{n}\right\}$, $v_{n}=\max \left\{v, \frac{1}{n}\right\}, \widetilde{w}_{n}=\min \{w, n\}$ and $\widetilde{v}_{n}=\min \{v, n\}$.

It is enough to prove (77) for bounded functions $f \in L^{p}(v)$ with compact support. It follows from the above remark that we may also assume that $w$ is locally integrable and there exists $\gamma>0$ such that

$$
0<\gamma \leq w(x) \text { for all } x \in \mathbb{R}^{2} .
$$

Let $E=\left\{x \in \mathbb{R}^{2}: \lambda<\mathcal{M}_{\xi}^{+2} f(x), \mathcal{M}^{+} f(x) \leq 2 \lambda\right\}$. We notice that the weighted measure $w(x) d x$ is finite on compact sets since $w$ is locally integrable. Therefore it is enough to show that there exists $C>0$ such that

$$
w(K) \leq \frac{C}{\lambda^{p}} \int_{\mathbb{R}^{2}}|f|^{p} v
$$

for all compact $K \subset E$.

Let us fix a compact set $K \subset E$. For each $x=\left(x_{1}, x_{2}\right) \in K$ there exists a square $Q_{x}=\left[x_{1}-l, x_{1}\right] \times\left[x_{2}-l, x_{2}\right]$ with $\xi \leq l$ and $l=2^{k}$ for some $k \in \mathbb{Z}$ such that

$$
\frac{\lambda}{4}<\frac{1}{\left|Q_{x}\right|} \int_{Q_{x}^{+2}}|f| .
$$

Let $Q_{x, 2 l}=\left[x_{1}, x_{1}+2 l\right] \times\left[x_{2}, x_{2}+2 l\right]$. It is clear that $\left(\widetilde{Q_{x}}\right)^{+2} \subset Q_{x, 2 l}$, and therefore

$$
\begin{aligned}
\frac{1}{\left|Q_{x}\right|} \int_{\left(\widetilde{Q_{x}}\right)^{+2}}|f| & \leq \frac{1}{\left|Q_{x}\right|} \int_{Q_{x, 2 l}}|f| \\
& =\frac{4}{\left|Q_{x, 2 l}\right|} \int_{Q_{x, 2 l}}|f| \leq 4 \mathcal{M}^{+} f(x) \leq 8 \lambda,
\end{aligned}
$$

where the last inequality follows from $x \in K \subset E$. Consequently, for each $x \in K$ we have a square $Q_{x}=\left[x_{1}-l, x_{1}\right] \times\left[x_{2}-l, x_{2}\right]$ such that $\xi \leq l$,

$$
\frac{\lambda}{4}<\frac{1}{\left|Q_{x}\right|} \int_{Q_{x}^{+2}}|f| \text { and } \frac{1}{\left|Q_{x}\right|} \int_{\left(\widetilde{Q_{x}}\right)^{+2}}|f| \leq 8 \lambda .
$$

Observe that $l \leq M$ for a certain positive real number $M$ depending on $\lambda$ and $f$. This follows from the inequalities

$$
\left|Q_{x}\right| \leq \frac{4}{\lambda} \int_{Q_{x}^{+2}}|f| \leq \frac{4}{\lambda} \int_{\mathbb{R}^{2}}|f|<\infty .
$$


Since $l \leq M, x \in K$ and $K$ is compact, we have that the union $\bigcup_{x \in K} \widetilde{Q_{x}}$ is a bounded set. Thus, there exists a square $R$ such that

$$
\bigcup_{x \in K} \widetilde{Q_{x}} \subset R
$$

Let us consider the square $2 R$. Since $w$ is integrable on $2 R$, there exists $\varepsilon, 0<\varepsilon<1$, such that if $Q \subset R$ is a square, then

$$
w((1+\varepsilon) Q \backslash Q) \leq \gamma \xi^{2} .
$$

If $l(Q) \geq \xi$ and $Q \subset R$, then

$$
w((1+\varepsilon) Q \backslash Q) \leq \gamma \xi^{2} \leq \gamma|Q| \leq w(Q) .
$$

Consequently,

$$
w((1+\varepsilon) Q) \leq 2 w(Q)
$$

for all squares $Q \subset R$ such that $l(Q) \geq \xi$. In particular

$$
w\left((1+\varepsilon) \widetilde{Q_{x}}\right) \leq 2 w\left(\widetilde{Q_{x}}\right), \quad \text { for all } x \in K .
$$

Let us denote by $B_{x}(r)$ the ball of center $x$ and radius $r$. It is clear that

$$
K \subset \bigcup_{x \in K} B_{x}\left(\frac{\xi \varepsilon}{2}\right) .
$$

Since $K$ is compact, there exist $x_{1}, \ldots, x_{s} \in K$ such that $K \subset \bigcup_{j=1}^{s} B_{x_{j}}\left(\frac{\xi \varepsilon}{2}\right)$. Applying the covering lemma to the set $A=\left\{x_{1}, \ldots, x_{s}\right\}$ and the squares $\left\{Q_{x_{j}}\right.$ : $j=1, \ldots, s\}$, there exists $\Gamma \subset\{1, \ldots, s\}$ such that

$$
A=\left\{x_{1}, \ldots, x_{s}\right\} \subset \bigcup_{i \in \Gamma} \widetilde{Q_{x_{i}}} .
$$

Further, there exists $\left\{F_{x_{i}}: i \in \Gamma\right\}$ such that

$$
\begin{gathered}
F_{x_{i}} \subset\left(\widetilde{Q_{x_{i}}}\right)^{+}, \\
\frac{\lambda}{8}<\frac{1}{\left|Q_{x_{i}}\right|} \int_{F_{x_{i}}}|f|,
\end{gathered}
$$

and

$$
\sum_{i \in \Gamma} \chi_{F_{x_{i}}}(x) \leq C
$$

Now, observe that if $x_{j} \in A$, then there exists $i \in \Gamma$ such that $x_{j} \in \widetilde{Q_{x_{i}}}$. This implies

$$
B_{x_{j}}\left(\frac{\xi \varepsilon}{2}\right) \subset(1+\varepsilon) \widetilde{Q_{x_{i}}}
$$

Therefore

$$
K \subset \bigcup_{j=1}^{s} B_{x_{j}}\left(\frac{\xi \varepsilon}{2}\right) \subset \bigcup_{i \in \Gamma}(1+\varepsilon) \widetilde{Q_{x_{i}}}
$$

and

$$
w(K) \leq \sum_{i \in \Gamma} w\left((1+\varepsilon) \widetilde{Q_{x_{i}}}\right) \leq 2 \sum_{i \in \Gamma} w\left(\widetilde{Q_{x_{i}}}\right)
$$


Assume now that $p>1$. Then using (15) and Hölder's inequality,

$$
\begin{aligned}
w(K) \leq 2 \sum_{i \in \Gamma} w\left(\widetilde{Q_{x_{i}}}\right) & \leq \frac{C}{\lambda^{p}} \sum_{i \in \Gamma} w\left(\widetilde{Q_{x_{i}}}\right)\left(\frac{1}{\left|Q_{x_{i}}\right|} \int_{F_{x_{i}}}|f|\right)^{p} \\
& =\frac{C}{\lambda^{p}} \sum_{i \in \Gamma} w\left(\widetilde{Q_{x_{i}}}\right)\left(\frac{1}{\left|Q_{x_{i}}\right|} \int_{F_{x_{i}}}|f| v^{\frac{1}{p}} v^{-\frac{1}{p}}\right)^{p} \\
& =\frac{C}{\lambda^{p}} \sum_{i \in \Gamma}\left[\frac{w\left(\widetilde{Q_{x_{i}}}\right)}{\left|Q_{x_{i}}\right|^{p}}\left(\int_{F_{x_{i}}} v^{-\frac{1}{p-1}}\right)^{p-1}\right] \int_{F_{x_{i}}}|f|^{p} v .
\end{aligned}
$$

Since $F_{x_{i}} \subset\left(\widetilde{Q_{x_{i}}}\right)^{+}$and from the $A_{p}^{+}\left(\mathbb{R}^{2}\right)$ condition we obtain

$$
\begin{aligned}
\frac{w\left(\widetilde{Q_{x_{i}}}\right)}{\left|Q_{x_{i}}\right|^{p}}\left(\int_{F_{x_{i}}} v^{-\frac{1}{p-1}}\right)^{p-1} & \leq \frac{w\left(\widetilde{Q_{x_{i}}}\right)}{\left|Q_{x_{i}}\right|^{p}}\left(\int_{\left(\widetilde{Q_{x_{i}}}\right)^{+}} v^{-\frac{1}{p-1}}\right)^{p-1} \\
& \leq C .
\end{aligned}
$$

Combining these last estimates and the fact that the sets $F_{x_{i}}, i \in \Gamma$, are almost disjoint, we get (13) for $p>1$.

If $p=1$ we use that

$$
\frac{w\left(\widetilde{Q_{x_{i}}}\right)}{\left|Q_{x_{i}}\right|} \leq C v(x)
$$

for almost every $x \in\left(\widetilde{Q_{x_{i}}}\right)+$ (by condition $A_{1}^{+}\left(\mathbb{R}^{2}\right)$ ). Since $F_{x_{i}} \subset\left(\widetilde{Q_{x_{i}}}\right)+$ we obtain

$$
\begin{aligned}
w(K) \leq 2 \sum_{i \in \Gamma} w\left(\widetilde{Q_{x_{i}}}\right) & \leq \frac{C}{\lambda} \sum_{i \in \Gamma} w\left(\widetilde{Q_{x_{i}}}\right) \frac{1}{\left|Q_{x_{i}}\right|} \int_{F_{x_{i}}}|f| \\
& \leq \frac{C}{\lambda} \sum_{i \in \Gamma} \int_{F_{x_{i}}}|f| v \\
& \leq \frac{C}{\lambda} \int_{\mathbb{R}^{2}}|f| v,
\end{aligned}
$$

where in the last inequality we have used that the sets $F_{x_{i}}$ are almost disjoint.

\section{Proof of Lemma 3.1}

Before starting with the proof of the covering lemma, we need to introduce a notion of maximality and to state some preliminary lemmas.

Definition 4.1. Assume that $\mathcal{F}$ is a finite family of squares. Let

$$
l_{0}=\max \{l(Q): Q \in \mathcal{F}\}
$$

and consider $\Sigma_{0}=\left\{Q \in \mathcal{F}: l(Q)=l_{0}\right\}$. Now consider $\mathcal{F}_{1}=\{Q \in \mathcal{F}: Q \cap R=$ $\emptyset$ for all $\left.R \in \Sigma_{0}\right\}$. If $\mathcal{F}_{1}=\emptyset$, then the process stops. If $\mathcal{F}_{1} \neq \emptyset$, then we take $l_{1}=$ $\max \left\{l(Q): Q \in \mathcal{F}_{1}\right\}$ and we set $\Sigma_{1}=\left\{Q \in \mathcal{F}_{1}: l(Q)=l_{1}\right\}$. Now we continue the process considering $\mathcal{F}_{2}=\left\{Q \in \mathcal{F}_{1}: Q \cap R=\emptyset\right.$ for all $\left.R \in \Sigma_{1}\right\}$. Since the family $F$ is finite the process stops in a finite number of steps. Assume that $\Sigma_{0}, \ldots, \Sigma_{k_{0}}$ have been chosen and $\mathcal{F}_{k_{0}+1}=\left\{Q \in \mathcal{F}_{k_{0}}: Q \cap R=\emptyset\right.$ for all $\left.R \in \Sigma_{k_{0}}\right\}=\emptyset$. We shall say that the squares belonging to $\bigcup_{i=0}^{k_{0}} \Sigma_{i}$ are maximal squares in $\mathcal{F}$. 
Remark 4.2. Observe that if $\mathcal{F}$ is a finite family of squares and $Q \in \mathcal{F}$, then either $Q$ is maximal in $\mathcal{F}$ or there exists a maximal square $Q_{m}$ such that $l(Q)<l\left(Q_{m}\right)$ and $Q_{m} \cap Q \neq \emptyset$. We also have that if $Q_{i}$ and $Q_{j}$ are maximal in $\mathcal{F}$, then $l\left(Q_{i}\right)=l\left(Q_{j}\right)$ or $Q_{i} \cap Q_{j}=\emptyset$.

Before stating the next lemma, we recall the notation introduced in $\S 2$. In particular, $\widetilde{Q}$ is the dilation of $Q$ to the right and to the bottom in half the length of the side of $Q$.

Lemma 4.3. Fix a square $Q$. Let $\mathcal{F}=\left\{Q_{j}: j \in \Gamma\right\}$ be a finite family of squares of dyadic size such that the squares $\widetilde{Q_{j}}, j \in \Gamma$, of the same size are almost disjoint, i.e., there exists a constant $A$ such that for all $l$

$$
\sum_{\left\{i \in \Gamma: l\left(Q_{i}\right)=l\right\}} \chi_{\widetilde{Q_{i}}} \leq A
$$

Let $\Gamma_{0}=\left\{j \in \Gamma:\left(\widetilde{Q_{j}} \cup\left(\widetilde{Q_{j}}\right)^{+}\right) \cap \partial Q \neq \emptyset,\left|Q_{j}\right|<|Q|\right\}$, where $\partial Q$ denotes the boundary of $Q$. Then there exists a constant $C$ depending only on $A$ such that

$$
\sum_{j \in \Gamma_{0}}\left|\widetilde{Q_{j}}\right| \leq C|Q|
$$

Proof. Let $l(Q)=l_{0}$. Then there exists $k_{0} \in \mathbb{Z}$ such that $2^{-k_{0}}<l_{0} \leq 2^{-k_{0}+1}$. Therefore

$$
\Gamma_{0}=\bigcup_{k=k_{0}}^{\infty}\left\{j \in \Gamma:\left(\widetilde{Q_{j}} \cup\left(\widetilde{Q_{j}}\right)^{+}\right) \cap \partial Q \neq \emptyset,\left|Q_{j}\right|=\left(1 / 2^{k}\right)^{2}\right\} .
$$

If $L_{i}, i=1,2,3,4$, are the sides of the square $Q$, it is clear that it will suffice to prove

$$
\sum_{k=k_{0}}^{\infty} \sum_{j \in \Gamma_{k, i}}\left|\widetilde{Q_{j}}\right| \leq C|Q|,
$$

where $\Gamma_{k, i}=\left\{j \in \Gamma:\left(\widetilde{Q_{j}} \cup\left(\widetilde{Q_{j}}\right)^{+}\right) \cap L_{i} \neq \emptyset,\left|Q_{j}\right|=\left(1 / 2^{k}\right)^{2}\right\}$. We will prove it for one of the sides, the argument being similar for the others. If $Q=[a, b] \times[c, d]$ let $L_{1}=\{a\} \times[c, d]$. Let $k \geq k_{0}$. Then there exists a rectangle $R_{k}$ such that $\left|R_{k}\right|=42 l_{0} 2^{-k}$ and

$$
\bigcup_{j \in \Gamma_{k, 1}} \widetilde{Q_{j}} \cup\left(\widetilde{Q_{j}}\right)^{+} \subset R_{k} .
$$

(Take $R_{k}=\left[a-\frac{3}{2^{k}}, a+\frac{3}{2^{k}}\right] \times\left[c-3 l_{0}, d+3 l_{0}\right]$.) Then

$$
\begin{aligned}
\sum_{j \in \Gamma_{k, 1}}\left|\widetilde{Q_{j}}\right| & =C \sum_{k=k_{0}}^{\infty} \sum_{j \in \Gamma_{k, 1}}\left|Q_{j}\right| \\
& \leq C \sum_{k=k_{0}}^{\infty}\left|\bigcup_{j \in \Gamma_{k, 1}} Q_{j}\right| \\
& \leq C \sum_{k=k_{0}}^{\infty}\left|R_{k}\right| \\
& \leq C \sum_{k=k_{0}}^{\infty} l_{0} 2^{-k}=C l_{0} 2^{-k_{0}} \leq C|Q|,
\end{aligned}
$$


where we have used in the first inequality the fact that the squares of equal size are almost disjoint.

Lemma 4.4. Fix a square $Q$. Let $\mathcal{F}=\left\{Q_{j}: j \in \Gamma\right\}$ be a finite family of squares of dyadic size such that the squares $\widetilde{Q_{j}}, j \in \Gamma$, of the same size are almost disjoint; i.e., there exists a constant $A$ such that for all $l$

$$
\sum_{\left\{i \in \Gamma: l\left(Q_{i}\right)=l\right\}} \chi_{\widetilde{Q_{i}}} \leq A .
$$

Assume that $\widetilde{Q_{j}}$ is not included in $\widetilde{Q_{i}}$ for any $j, i \in \Gamma, j \neq i$. Let $\Gamma_{0}=\{j \in \Gamma$ : $\left.\left(\widetilde{Q_{j}}\right)^{+} \cap(\widetilde{Q})^{+} \neq \emptyset,\left|Q_{j}\right|<|Q|\right\}$. Then there exists a constant $C$ depending only on A such that

$$
\sum_{j \in \Gamma_{0}}\left|\widetilde{Q_{j}}\right| \leq C|Q|
$$

Proof. Let $\Gamma_{1}=\left\{j \in \Gamma_{0}:\left(\widetilde{Q_{j}} \cup\left(\widetilde{Q_{j}}\right)^{+}\right) \cap \partial(\widetilde{Q})^{+} \neq \emptyset\right\}$ and $\Gamma_{2}=\left\{j \in \Gamma_{0}:\right.$ $\left.\widetilde{Q_{j}} \cup\left(\widetilde{Q_{j}}\right)^{+} \subset(\widetilde{Q})^{+}\right\}$. It is clear that $\Gamma_{0}=\Gamma_{1} \cup \Gamma_{2}$. Then, applying Lemma 4.3 with $Q=(\widetilde{Q})^{+}$, we can see that $\sum_{j \in \Gamma_{1}}\left|\widetilde{Q}_{j}\right| \leq C|Q|$. Now, we will see that $\sum_{j \in \Gamma_{2}}\left|\widetilde{Q_{j}}\right| \leq C|Q|$. We consider the maximal squares $\widetilde{Q_{i}}$ in the sense of Definition 4.1 for the family $\left\{\widetilde{Q_{j}}: j \in \Gamma_{2}\right\}$. Then

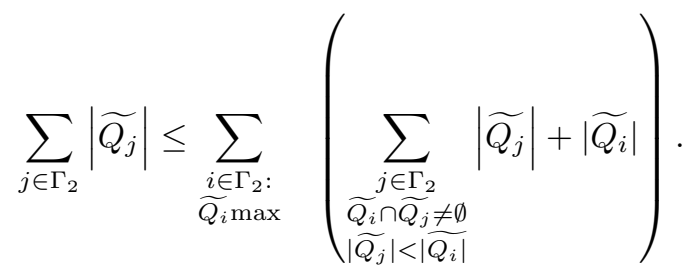

Since $\widetilde{Q_{j}}$ is not included in $\widetilde{Q_{i}}$, we have that

$$
\left\{j \in \Gamma_{2}: \widetilde{Q_{j}} \cap \widetilde{Q_{i}} \neq \emptyset,\left|\widetilde{Q_{j}}\right|<\left|\widetilde{Q_{i}}\right|\right\}=\left\{j \in \Gamma_{2}: \widetilde{Q_{j}} \cap \partial \widetilde{Q_{i}} \neq \emptyset,\left|\widetilde{Q_{j}}\right|<\left|\widetilde{Q_{i}}\right|\right\} .
$$

Now, applying Lemma 4.3 with $Q=\widetilde{Q_{i}}$, we get

$$
\sum_{\substack{j \in \Gamma_{2} \\ \widetilde{Q_{i}} \cap \widetilde{Q_{j}} \neq \emptyset \\\left|\widetilde{Q_{j}}\right|<\left|\widetilde{Q_{i}}\right|}}\left|\widetilde{Q_{j}}\right| \leq C\left|\widetilde{Q_{i}}\right| .
$$

Since the squares $\widetilde{Q_{i}}$ are almost disjoint and $\widetilde{Q_{i}} \subset(\widetilde{Q})^{+}$, we obtain that

$$
\begin{aligned}
\sum_{j \in \Gamma_{2}}\left|\widetilde{Q_{j}}\right| & \leq C \sum_{\substack{i \in \Gamma_{2}: \\
\widetilde{Q}_{i} \max }}\left|\widetilde{Q_{i}}\right| \\
& \leq C\left|(\widetilde{Q})^{+}\right|=C|Q| .
\end{aligned}
$$

Proof of Lemma 3.1. We shall do two selections.

First selection: Let $B_{1}$ be the set of points of $A$ such that their second coordinate is the largest one among the second coordinates of the points in $A$. Let 
$x_{i_{1}}$ be the point of $B_{1}$ with the smallest first coordinate. Assume that $x_{i_{1}}, \ldots, x_{i_{k}}$ have been chosen. We define $A_{k+1}=A \backslash \bigcup_{j=1}^{k} \widetilde{Q_{i_{j}}}$. If $A_{k+1}=\emptyset$, then we do not choose more points. If $A_{k+1} \neq \emptyset$, then we consider the set $B_{k+1}$ of points of $A_{k+1}$ such that the second coordinate is the largest one among the second coordinates of the points in $A_{k+1}$ and we choose $x_{i_{k+1}}$ as the point in $B_{k+1}$ with the smaller first coordinate. Since we have a finite number of squares the process stops. Let $\Delta=\left\{i: x_{i}\right.$ was chosen at some moment $\}$. Then we have the following properties:

(a) $A \subset \bigcup_{i \in \Delta} \widetilde{Q_{i}}$.

(b) $\frac{1}{\left|Q_{i}\right|} \int_{\left(\widetilde{Q_{i}}\right)^{+}} f>\frac{\lambda}{4}$ for all $i \in \Delta$.

(c) If $l>0$ and $Q_{j}$ and $Q_{k}$ are two squares with $j, k \in \Delta$ and $l\left(Q_{j}\right)=l\left(Q_{k}\right)=l$, then the norm $\left\|x_{j}-x_{k}\right\|_{\infty}$ is greater than $l / 2$.

(d) The squares $\widetilde{Q_{i}}, i \in \Delta$, of the same size are almost disjoint; i.e., there exists a constant $C$ such that for all $l$

$$
\sum_{\left\{i \in \Delta: l\left(Q_{i}\right)=l\right\}} \chi_{\widetilde{Q_{i}}} \leq C
$$

$(C=36$ is valid).

Property (a) is clear. In fact, if $A$ is not included in $\bigcup_{i \in \Delta} \widetilde{Q_{i}}$, then $A \backslash \bigcup_{i \in \Delta} \widetilde{Q_{i}} \neq \emptyset$ and the process would continue. Property (b) follows from $Q_{i}^{+2} \subset\left(\widetilde{Q}_{i}\right)^{+}$and the assumption in the lemma. In order to see that property (c) holds we may assume that the point $x_{k}$ was chosen before $x_{j}$. Then (c) follows since the second coordinate of $x_{k}$ is greater than or equal to the second coordinate of $x_{j}, x_{j} \notin \widetilde{Q_{k}}$ and $l\left(Q_{k}\right)=l$ (see Figures 3 and 4 ).

Finally, property (d) follows from (c). Let us fix a square $\widetilde{Q_{j_{0}}}=\left[z_{j_{0}}-\frac{3}{2} l, z_{j_{0}}\right] \times$ $\left[y_{j_{0}}-\frac{3}{2} l, y_{j_{0}}\right]$ of the selection, i.e., $\left(z_{j_{0}}, y_{j_{0}}\right)$ is the upper-right corner. Then if $\widetilde{Q_{j}}$ is a square of the selection with $l\left(\widetilde{Q_{j}}\right)=\frac{3}{2} l$ and $\widetilde{Q_{j}} \cap \widetilde{Q_{j_{0}}} \neq \emptyset$, then the upper-right corner $\left(z_{j}, y_{j}\right)$ of $\widetilde{Q_{j}}$ belongs to the square $R_{j_{0}}=\left[z_{j_{0}}-\frac{3}{2} l, z_{j_{0}}+\frac{3}{2} l\right] \times$ $\left[y_{j_{0}}-\frac{3}{2} l, y_{j_{0}}+\frac{3}{2} l\right]$. Now, we divide $R_{j_{0}}$ in 36 disjoint and equal squares of size $\frac{l}{2}$. The point $\left(z_{j}, y_{j}\right)$ belongs to only one of those squares. Therefore, it follows from (c) that there are not more than 36 squares $\widetilde{Q_{j}}$ in the collection (one of them is $\left.\widetilde{Q_{j_{0}}}\right)$ of size $l\left(\widetilde{Q_{j}}\right)=l\left(\widetilde{Q_{j_{0}}}\right)$ that intersect $\widetilde{Q_{j_{0}}}$. This proves (d).

Second selection: Let $\Gamma_{1}=\Delta$. We take $\widetilde{Q_{i_{1}}} \in\left\{\widetilde{Q_{i}}: i \in \Gamma_{1}\right\}$ such that $l\left(\widetilde{Q_{i_{1}}}\right)=\max \left\{l\left(\widetilde{Q_{i}}\right): i \in \Gamma_{1}\right\}$. Once we have chosen $\widetilde{Q_{i_{1}}}, \ldots, \widetilde{Q_{i_{k}}}$, we consider

$$
\Gamma_{k+1}=\left\{i \in \Delta: \text { such that } \widetilde{Q_{i}} \text { is not contained in } \widetilde{Q_{i_{l}}} \text { for } l=1, \ldots, k\right\} .
$$

If $\Gamma_{k+1}=\emptyset$ we do not choose more squares. If $\Gamma_{k+1} \neq \emptyset$ we choose $\widetilde{Q_{i_{k+1}}} \in\left\{\widetilde{Q_{i}}: i \in\right.$ $\left.\Gamma_{k+1}\right\}$ such that $l\left(\widetilde{Q_{i_{k+1}}}\right)=\max \left\{l\left(\widetilde{Q_{i}}\right): i \in \Gamma_{k+1}\right\}$. Since we have a finite number of squares, the process stops in a finite number of steps. Let

$$
\Gamma=\left\{i \in \Delta: \text { such that } \widetilde{Q_{i}} \text { was chosen in one of the steps above }\right\} .
$$

The family $\left\{\widetilde{Q_{i}}: i \in \Gamma\right\}$ has the properties (a)-(d), and it also satisfies the following property:

(e) If $i, j \in \Gamma, i \neq j$, then $\widetilde{Q_{j}} \not \widetilde{Q_{i}}$. 


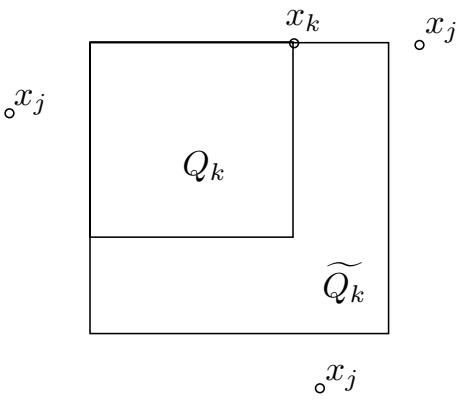

Figure 3. Possible places for $x_{j}$ if $x_{k}$ was chosen before $x_{j}$.

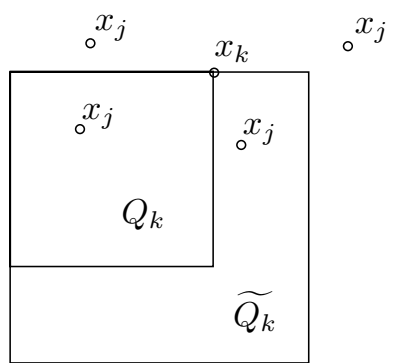

Figure 4. Impossible places for $x_{j}$ if $x_{k}$ was chosen before $x_{j}$.

We have already proved the first part of the lemma. Now we assume

$$
\frac{1}{\left|Q_{j}\right|} \int_{\left(\widetilde{Q_{j}}\right)^{+}} f \leq 8 \lambda
$$

and we proceed to select the family $\left\{F_{j}\right\}$. We can apply Lemma 4.4 to the family $\left\{Q_{j}: j \in \Gamma\right\}$. For fixed $j \in \Gamma$ and $\Gamma_{j}=\left\{i \in \Gamma:\left(\widetilde{Q_{i}}\right)^{+} \cap\left(\widetilde{Q_{j}}\right)^{+} \neq \emptyset,\left|Q_{i}\right|<\left|Q_{j}\right|\right\}$ we have by Lemma 4.4 that

$$
\sum_{i \in \Gamma_{j}}\left|Q_{i}\right| \leq \sum_{i \in \Gamma_{j}}\left|\widetilde{Q_{i}}\right| \leq C\left|Q_{j}\right|
$$

Consequently,

$$
\begin{aligned}
\sum_{i \in \Gamma_{j}} \int_{\left(\widetilde{Q_{i}}\right)^{+}} f & \leq 8 \lambda \sum_{i \in \Gamma_{j}}\left|Q_{i}\right| \\
& \leq 8 C \lambda\left|Q_{j}\right| \\
& <32 C \int_{\left(\widetilde{Q_{j}}\right)^{+}} f .
\end{aligned}
$$

Therefore, we have proved that there exists a natural number $N$ such that

$$
\sum_{i \in \Gamma_{j}} \int_{\left(\widetilde{Q_{i}}\right)^{+}} f \leq N \int_{\left(\widetilde{Q_{j}}\right)^{+}} f
$$


where $N$ is independent of $f, \lambda$ and $j$. Let $s$ be the number of elements of $\Gamma$. If $s \leq 2 N$ we choose $F_{j}=\left(\widetilde{Q_{j}}\right)^{+}$, and there is nothing to prove. Suppose $s>2 N$. We define for each $n, 1 \leq n \leq s$, a subset $E_{n}^{j}$ of $\left(\widetilde{Q_{j}}\right)^{+}$, in the following way:

$$
E_{n}^{j}=\left\{x \in\left(\widetilde{Q_{j}}\right)^{+}: \text {there exist at least } n \text { numbers } i \in \Gamma\right.
$$

$$
\text { such that } \left.x \in\left(\widetilde{Q_{i}}\right)^{+} \text {and }\left|Q_{i}\right|<\left|Q_{j}\right|\right\} \text {. }
$$

Clearly $E_{n+1}^{j} \subset E_{n}^{j}$. Moreover, for $x \in\left(\widetilde{Q_{j}}\right)^{+}$

$$
\sum_{n=1}^{s} \chi_{E_{n}^{j}}(x) \leq \sum_{i \in \Gamma_{j}} \chi_{\left(\widetilde{Q_{i}}\right)^{+}}(x) .
$$

In fact, if $\sum_{n=1}^{s} \chi_{E_{n}^{j}}(x)=k$, then $x \in\left(\widetilde{Q_{i}}\right)^{+}$for $k$ indexes $i$ with $\left|Q_{i}\right|<\left|Q_{j}\right|$. But these $k$ indexes belong to $\Gamma_{j}$; therefore $\sum_{i \in \Gamma_{j}} \chi_{\left(\widetilde{Q_{i}}\right)^{+}}(x) \geq k$ and the inequality is true. Now, by (18) and (19) we get

$$
\begin{aligned}
\sum_{n=1}^{s} \int_{E_{n}^{j}} f(x) d x & \leq \int_{\left(\widetilde{Q_{j}}\right)^{+}} f(x)\left(\sum_{i \in \Gamma_{j}} \chi_{\left(\widetilde{Q_{i}}\right)^{+}}(x)\right) d x \\
& \leq \sum_{i \in \Gamma_{j}} \int_{\left(\widetilde{Q_{i}}\right)^{+}} f \leq N \int_{\left(\widetilde{Q_{j}}\right)^{+}} f .
\end{aligned}
$$

This inequality, $s>2 N$ and $\int_{E_{n+1}^{j}} f \leq \int_{E_{n}^{j}} f$ give

$$
2 N \int_{E_{2 N}^{j}} f \leq \sum_{n=1}^{s} \int_{E_{n}^{j}} f \leq N \int_{\left(\widetilde{Q_{j}}\right)^{+}} f .
$$

Therefore,

$$
\int_{E_{2 N}^{j}} f \leq \frac{1}{2} \int_{\left(\widetilde{Q_{j}}\right)^{+}} f
$$

From this last inequality, if we define $F_{j}=\left(\widetilde{Q_{j}}\right)^{+}-E_{2 N}^{j}$, we get

Thus

$$
\int_{F_{j}} f \geq \frac{1}{2} \int_{\left(\widetilde{Q_{j}}\right)^{+}} f
$$

$$
\frac{1}{\left|Q_{j}\right|} \int_{F_{j}} f \geq \frac{1}{2\left|Q_{j}\right|} \int_{\left(\widetilde{Q_{j}}\right)^{+}} f>\frac{\lambda}{8}
$$

It only remains to prove that the sets $F_{j}$ are almost disjoint. We will prove

$$
\sum_{j \in \Gamma} \chi_{F_{j}}(x) \leq 72 N
$$

Let $x \in \bigcap_{i=1}^{k} F_{j_{i}}$, with $j_{i} \in \Gamma$. We will show that $k \leq 72 N$. Since $F_{j_{i}} \subset\left(\widetilde{Q_{j_{i}}}\right)^{+}$ we have $x \in\left(\widetilde{Q_{j_{i}}}\right)^{+}$. Consider all the squares $\left(\widetilde{Q_{j_{i}}}\right)^{+}$of maximum radius (there cannot be more than 36). For any of them (call it $\left(\widetilde{Q_{j_{0}}}\right)^{+}$) there can be no more than $2 N$ of smaller size such that $x$ belongs to those squares. Suppose this were not true. Then $x$ belongs to $s$ squares with $s>2 N$ of size smaller than $\left(\widetilde{Q_{j_{i_{0}}}}\right)^{+}$ and therefore $x \in E_{s}^{j_{i_{0}}} \subset E_{2 N}^{j_{i_{0}}}$, a contradiction since $x \in F_{j_{i_{0}}}=\left(\widetilde{Q_{j_{i_{0}}}}\right)^{+}-E_{2 N}^{j_{i_{0}}}$. Therefore (20) follows. 


\section{Proof of Theorem [.5]}

To prove Theorem 1.5 we need to recall some facts and to introduce some notation. For each $t \in \mathbb{R}^{2}$ we consider the measures $\mu_{t}$ defined by

$$
\mu_{t}(E)=\mu\left(\tau^{t}(E)\right)
$$

These measures have the same sets of measure zero as $\mu$ since the transformations are null-preserving. If $H_{t}$ is the Radon-Nikodym derivative of $\mu_{t}$ with respect to $\mu$, then

and

$$
\mu(E)=\int_{X}\left(T^{t} \chi_{E}\right) H_{t} d \mu
$$

$$
H_{t+s}=\left(T^{t} H_{s}\right) H_{t} .
$$

It follows that the operators $S^{t} f=H_{t} T^{t} f$ are isometries in $L^{1}(\mu)$. Consequently, by using [6, Lemma III.11.16] we may assume without loss of generality that $H_{t}(x)$ is measurable with respect to the completion of the $\sigma$-algebra product (see also [15]).

Proof of Theorem 1.5. Since $\mathcal{T}$ is Cesàro bounded in $L^{1}(\mu)$, we have by Tonelli's theorem that

$$
\frac{1}{h^{2}} \int_{0}^{h} \int_{0}^{h} \int_{X} T^{t-s} f(x) d \mu d t \leq C \int_{X} T^{-s} f(x) d \mu
$$

for every $h>0$, all $s=\left(s_{1}, s_{2}\right) \in \mathbb{R}^{2}$ and each measurable function $f \geq 0$. But

$$
\int_{X} T^{t} f(x) d \mu=\int_{X} f(x) H_{-t}(x) d \mu .
$$

Therefore

$$
\int_{X} f(x)\left(\frac{1}{h^{2}} \int_{0}^{h} \int_{0}^{h} H_{s-t}(x) d t\right) d \mu \leq C \int_{X} f(x) H_{s}(x) d \mu
$$

for all nonnegative measurable functions $f$, which implies

$$
\frac{1}{h^{2}} \int_{s_{1}-h}^{s_{1}} \int_{s_{2}-h}^{s_{2}} H_{t}(x) d t d \mu \leq C H_{s}(x) \quad \text { a.e. } x \text {. }
$$

It follows that for almost every $x \in X$

$$
\frac{1}{h^{2}} \int_{s_{1}-h}^{s_{1}} \int_{s_{2}-h}^{s_{2}} H_{t}(x) d t d \mu \leq C H_{s}(x) \text { for a.e. } s=\left(s_{1}, s_{2}\right),
$$

or, in other words, for almost every $x$ the functions $t \rightarrow H_{t}(x)$ satisfy $A_{1}^{+}\left(\mathbb{R}^{2}\right)$ with a constant independent of $x$. Now we obtain the weak type $(1,1)$ inequality by transference arguments.

We can assume that $f \geq 0$. For each $\eta>0$, let us consider $M_{\eta}^{+} f(x)=$ $\sup _{0<h \leq \eta} A_{h} f(x)$. Let $\lambda>0$ and $E_{\lambda}=\left\{x \in X: M_{\eta}^{+} f(x)>\lambda\right\}$. Let us fix $R>0$. Then

$$
\begin{aligned}
\mu\left(E_{\lambda}\right) & =\frac{1}{R^{2}} \int_{0}^{R} \int_{0}^{R} \int_{X} T^{t} \chi_{E_{\lambda}}(x) H_{t}(x) d \mu(x) d t \\
& =\int_{X} \frac{1}{R^{2}} \int_{0}^{R} \int_{0}^{R} T^{t} \chi_{E_{\lambda}}(x) H_{t}(x) d \mu(x) d t .
\end{aligned}
$$


If we define $g^{x}(t)=T^{t} g(x)$, we have that if $R>0, t=\left(t_{1}, t_{2}\right), t_{1} \leq R, t_{2} \leq R$ and $T^{t} \chi_{E_{\lambda}}(x)=1$, then $M^{+}\left(f^{x} \chi_{[0, R+\eta] \times[0, R+\eta]}\right)(t)>\lambda$. Therefore

$$
\mu\left(E_{\lambda}\right) \leq \int_{X} \frac{1}{R^{2}} \int_{\left\{t: M^{+}\left(f^{x} \chi_{[0, R+\eta] \times[0, R+\eta]}\right)(t)>\lambda\right\}} H_{t}(x) d \mu(x) d t .
$$

Since, for almost every $x$, the functions $t \rightarrow H_{t}(x)$ satisfy $A_{1}^{+}\left(\mathbb{R}^{2}\right)$ with a constant independent of $x$, we obtain by Theorem 1.2 that the last term is dominated by

$$
\begin{aligned}
\frac{C}{\lambda} \int_{X} \frac{1}{R^{2}} \int_{0}^{R+\eta} \int_{0}^{R+\eta} & f^{x}(t) H_{t}(x) d t d \mu(x) \\
& =\frac{C}{\lambda} \frac{1}{R^{2}} \int_{0}^{R+\eta} \int_{0}^{R+\eta} \int_{X} T^{t} f(x) H_{t}(x) d \mu(x) d t \\
& =\frac{C}{\lambda} \frac{1}{R^{2}} \int_{0}^{R+\eta} \int_{0}^{R+\eta} \int_{X} f(x) d \mu(x) d t \\
& =\frac{C}{\lambda}\left(\frac{R+\eta}{R}\right)^{2} \int_{X} f(x) d \mu(x) .
\end{aligned}
$$

Letting $R$ go to infinity we obtain

$$
\mu\left(E_{\lambda}\right) \leq \frac{C}{\lambda} \int_{X} f(x) d \mu(x)
$$

Letting $\eta$ tend to infinity we obtain the inequality that we wished to prove.

Final Remarks 5.1. It is clear that $M_{\mathcal{T}}$ is bounded in $L^{\infty}(\mu)$. Therefore, under the assumptions in Theorem 1.5 we have that $M_{\mathcal{T}}$ is of strong type $(p, p)$ for all $p>1$.

It is worth noting that there exist flows which are Cesàro bounded in $L^{1}(\mu)$. In order to see this, consider a flow of measure-preserving transformations, that is, $\mu\left(\tau^{t} E\right)=\mu(E)$. Let us take the ergodic maximal operator

$$
\mathcal{N}_{\mathcal{T}} f(x)=\sup _{h>0} \frac{1}{h^{2}}\left|\int_{0}^{h} \int_{0}^{h} f\left(\tau^{-t} x\right) d t\right| .
$$

It is known that $\mathcal{N}_{\mathcal{T}}$ is of weak type $(1,1)$ and of strong type $(p, p)$ for $p>1$. Fix $p, 1<p<\infty$, and a positive function $g \in L^{p}(\mu)$. Let $A$ be a constant such that

$$
\left\|\mathcal{N}_{\mathcal{T}} f\right\|_{L^{p}(\mu)} \leq A\|f\|_{L^{p}(\mu)}
$$

and define

$$
w=\sum_{i=0}^{\infty} \frac{\mathcal{N}_{\mathcal{T}}^{(i)} g}{(2 A)^{i}},
$$

where $\mathcal{N}_{\mathcal{T}}^{(i)}$ is the $i$ th-iteration of $\mathcal{N}_{\mathcal{T}}$. It is clear that $w \in L^{p}(\mu),\|w\|_{L^{p}(\mu)} \leq$ $2\|g\|_{L^{p}(\mu)}, g \leq w$ and $\mathcal{N}_{\mathcal{T}} w \leq 2 A w$ a.e. Now consider the measure $\tilde{\mu}=w d \mu$. The last property of $w$ implies that the flow is Cesàro bounded in $L^{1}(\tilde{\mu})$, and it is clear that the transformations $\tau^{t}$ are null-preserving transformations with respect to $\tilde{\mu}$. Further, if we have that for some $t \in \mathbb{R}^{2}$ the transformation $\tau^{t}$ is ergodic and the function $g \notin L^{\infty}(\mu)$, then the operators $T^{t}$ are not contractions. Moreover, they are not power bounded, that is, there is not a positive constant such that for all $t$

$$
\int_{X}\left|T^{t} f\right| d \tilde{\mu} \leq C \int_{X} f d \tilde{\mu}
$$


A more detailed discussion in the one-dimensional case for the two-sided case can be found in 9 .

Finally, we point out that Theorem [1.5] remains true for $1<p<\infty$. That is, if the group $\mathcal{T}$ is Cesàro bounded in $L^{p}(\mu), 1<p<\infty$, which means that there exists $C$ such that

$$
\sup _{h>0} \int_{X}\left|A_{h} f\right|^{p} d \mu \leq C \int_{X}|f|^{p} d \mu
$$

for all measurable functions $f \geq 0$, then there is a positive constant $C$ such that

$$
\mu\left(\left\{x \in X: M_{\mathcal{T}} f(x)>\lambda\right\}\right) \leq \frac{C}{\lambda^{p}} \int_{X}|f|^{p} d \mu
$$

for all $\lambda>0$ and all $f \in L^{p}(\mu)$. The weak type inequality follows by transference arguments, as in the case $p=1$, by using the fact that the functions $t \rightarrow H_{t}(x)$ satisfy $A_{p}^{+}\left(\mathbb{R}^{2}\right)$ for almost every $x$ with a uniform constant. All we have to show is that (25) implies that the functions $t \rightarrow H_{t}(x)$ satisfy $A_{p}^{+}\left(\mathbb{R}^{2}\right)$ for almost every $x$. The proof is similar to the one-dimensional case [3] - it uses the ideas of the factorization of weights [5, 7, but is not as direct as the corresponding one for $p=1$.

\section{REFERENCES}

[1] K. Andersen and B. Muckenhoupt, Weighted weak type Hardy inequalities with applications to Hilbert transforms and maximal functions, Studia Math. 72 (1982), no. 1, 9-26. MR665888 (83k:42018)

[2] E. Berkson and T.A. Gillespie, Mean-boundedness and Littlewood-Paley for separationpreserving operators, Trans. Amer. Math. Soc. 349 (1997), no. 3, 1169-1189. MR.1407694 (98b:42011)

[3] A.L. Bernardis, M. Lorente, F.J. Martín-Reyes, M.T. Martínez and A. de la Torre, Differences of ergodic averages for Cesàro bounded operators, Q. J. Math. 58 (2007), no. 2, 137-150. MR2334858 (2009g:47027)

[4] R. Coifman and C. Fefferman, Weighted norm inequalities for maximal functions and singular integrals, Studia Math. 51 (1974), 241-250. MR0358205 (50:10670)

[5] R. Coifman, P.W. Jones and J.L. Rubio de Francia, Constructive decomposition of BMO functions and factorization of $A_{p}$ weights, Proc. Amer. Math. Soc. 87 (1983), no. 4, 675-676. MR687639 (84c:42031)

[6] N. Dunford and J.T. Schwartz, Linear Operators. I, Pure and Applied Mathematics, Vol. 7 Interscience Publishers, Inc., New York; Interscience Publishers, Ltd., London 1958. MR0117523 (22:8302)

[7] J. García-Cuerva and J.L. Rubio de Francia, Weighted norm inequalities and related topics, North-Holland Mathematics Studies, 116. Notas de Matemática [Mathematical Notes], 104. North-Holland Publishing Co., Amsterdam, 1985. MR807149 (87d:42023)

[8] T.A. Gillespie and J.L. Torrea, Weighted ergodic theory and dimension free estimates., Q. J. Math. 54 (2003), no. 3, 257-280. MR2013139 (2004k:47015)

[9] M. Lorente and F.J. Martín-Reyes, The ergodic Hilbert transform for Cesàro bounded flows, Tohoku Math. J. (2) 46 (1994), no. 4, 541-556. MR.1301288 (95k:28037)

[10] F.J. Martín-Reyes, On the one-sided Hardy-Littlewood maximal function in the real line and in dimensions greater than one, Fourier analysis and partial differential equations (Miraflores de la Sierra, 1992), 237-250, Stud. Adv. Math., CRC, Boca Raton, FL, 1995. MR.1330244 (96f:42023)

[11] F.J. Martín-Reyes and A. de la Torre, The dominated ergodic estimate for mean bounded, invertible, positive operators, Proc. Amer. Math. Soc. 104 (1988), no. 1, 69-75. MR958045 (89i:47015)

[12] B. Muckenhoupt, Weighted norm inequalities for the Hardy maximal function, Trans. Amer. Math. Soc. 165 (1972), 207-226. MR0293384(45:2461) 
[13] S. Ombrosi, Weak weighted inequalities for a dyadic one-sided maximal function in $\mathbb{R}^{n}$, Proc. Amer. Math. Soc. 133 (2005), no. 6, 1769-1775. MR2120277(2005k:42055)

[14] R. Sato, A remark on the ergodic Hilbert transform, Math. J. Okayama Univ. 28 (1986), 159-163 (1987). MR 885025 (88k:47043)

[15] R. Sato, An ergodic maximal equality for nonsingular flows and applications, Acta Math. Hungar. 61 (1993), no. 1-2, 51-58. MR.1200957 (94c:28020)

[16] E. Sawyer, Weighted inequalities for the two-dimensional Hardy operator, Studia Math. 82 (1985), no. 1, 1-16. MR809769 (87f:42052)

[17] E. Sawyer, Weighted inequalities for the one-sided Hardy-Littlewood maximal functions, Trans. Amer. Math. Soc. 297 (1986), no. 1, 53-61. MR849466 (87k:42018)

[18] E. Stein and R. Shakarchi, Real analysis. Measure theory, integration, and Hilbert spaces, Princeton Lectures in Analysis, III. Princeton University Press, Princeton, NJ, 2005. MR2129625 (2005k:28024)

IMAL-CONICET, Facultad de Ingeniería Química, Universidad Nacional del Litoral, Güemes 3450, 3000 Santa Fe, Argentina

E-mail address: liliana.forzani@gmail.com

Departamento de Análisis Matemático, Facultad de Ciencias, Universidad de Málaga, 29071-MÁLAGA, SPAIN

E-mail address: martin_reyes@uma.es

Departamento de Matemática, Universidad Nacional del Sur, Bahía Blanca, 8000, Argentina

E-mail address: sombrosi@uns.edu.ar 